Supporting Information

\title{
Wetting Transition on Liquid-Repellent Surfaces Probed by Surface Force Measurements and Confocal Imaging
}

Mimmi Eriksson ${ }^{\dagger *}$, Per Martin Claesson ${ }^{\dagger+}$, Mikael Järn ${ }^{\dagger}$, Mikko Tuominen $^{\dagger}$, Viveca Wallqvist $^{\dagger}$, Joachim Schoelkopf ${ }^{\S}$, Patrick A.C. Gane ", and Agne Swerin ${ }^{\not}$

${ }^{\dagger}$ RISE Research Institutes of Sweden, SE-11486 Stockholm, Sweden

* KTH Royal Institute of Technology, School of Engineering Sciences in Chemistry, Biotechnology and Health, Department of Chemistry, Division of Surface and Corrosion Science, SE-10044 Stockholm, Sweden

$\S$ Omya International AG, CH-4665 Oftringen, Switzerland

" Aalto University, School of Chemical Engineering, Department of Bioproducts and Biosystems, FI-00076 Aalto, Finland

*Corresponding author: mimmi.eriksson@ri.se 


\section{Hydrodynamic force}

The hydrodynamic force $F_{\mathrm{H}}$ when a spherical particle of radius $R$ is approaching a flat surface with a velocity $v$ depends on the separation distance $D$, and can be estimated by: ${ }^{1}$

$$
F_{\mathrm{H}}=\frac{6 \pi \eta v R^{2}}{D}
$$

Eq. S1 is assuming a non-slip boundary condition, a Newtonian liquid with viscosity $\eta$ and is valid for small distances $(D<<R)$. However, the non-slip assumption is generally not valid, and it has been shown that that slip occurs for water on hydrophobic surfaces. ${ }^{2-4}$ To take the slip at solid surfaces into account a correction factor $f^{*}$ can be introduced to the equation for the hydrodynamic force:

$$
F_{\mathrm{H}}=\frac{6 \pi \eta v R^{2}}{D} f^{*}
$$

where $f^{*}$ depends on the slip length $b$ and the separation distance $D$ between the interacting surfaces, and for two hydrophobic surfaces is defined as: ${ }^{2}$

$$
f^{*}=\frac{D}{3 b}\left[\left(1+\frac{D}{6 b}\right) \ln \left(1+\frac{6 b}{D}\right)-1\right]
$$

Typical slip lengths on hydrophobic and superhydrophobic surfaces have been reported to be in the range of $40-260 \mathrm{~nm} .^{4,5}$

If we estimate the hydrodynamic force using Eq. S2 and Eq. S3 for our system $(R=3 \mu \mathrm{m}, v=$ $200 \mathrm{~nm} \mathrm{~s}^{-1}, \eta=8.9 \times 10^{-4} \mathrm{~Pa} \mathrm{~s}$ (water), $b=40 \mathrm{~nm}$ ), and for small separation $(D<10 \mathrm{~nm})$, we find $F_{\mathrm{H}}$ to be in the order of $10^{-12} \mathrm{~N}$. This is three orders of magnitude smaller than our smallest measured forces $\left(10^{-9} \mathrm{~N}\right)$, and thus we can ignore the hydrodynamic force. 


\section{Optical interference}

To adjust for interference patterns in the force curves, the zero force line was fitted to a sine function:

$$
F=A \sin \left(\frac{2 \pi}{P}(D+\varphi)\right)+C
$$

The fitting parameters amplitude $A$, periodicity $P$, phase shift $\varphi$ and vertical shift $C$ were extracted from the fitted function. The optical interference is pronounced for the superhydrophobic surface in water and 20 vol\% ethanol where an air layer is observed by confocal imaging (Figure S2). Interference is also observed in 40 vol\% ethanol where the resolution of the confocal image does not allow detection of an air layer (Figure S2). Some force curves where no attraction was observed are illustrated in Figure S3, and here we note the optical interference. Fits of Eq. 4 to the optical interference observed are illustrated in Figure S4.

\section{References}

1. Butt, H. J.; Cappella, B.; Kappl, M., Force Measurements with the Atomic Force Microscope: Technique, Interpretation and Applications. Surf. Sci. Rep. 2005, 59, 1-152.

2. Vinogradova, O. I., Drainage of a Thin Liquid Film Confined between Hydrophobic Surfaces. Langmuir 1995, 11, 2213-2220.

3. Vinogradova, O. I., Slippage of Water over Hydrophobic Surfaces. Int. J. Miner. Process. 1999, 56, 31-60.

4. Bhushan, B.; Wang, Y.; Maali, A., Boundary Slip Study on Hydrophilic, Hydrophobic, and Superhydrophobic Surfaces with Dynamic Atomic Force Microscopy. Langmuir 2009, 25, 8117-8121.

5. Wang, Y.; Bhushan, B.; Maali, A., Atomic Force Microscopy Measurement of Boundary Slip on Hydrophilic, Hydrophobic, and Superhydrophobic Surfaces. J. Vac. Sci. Technol., A 2009, 27, 754-760. 


\section{Supporting figures}
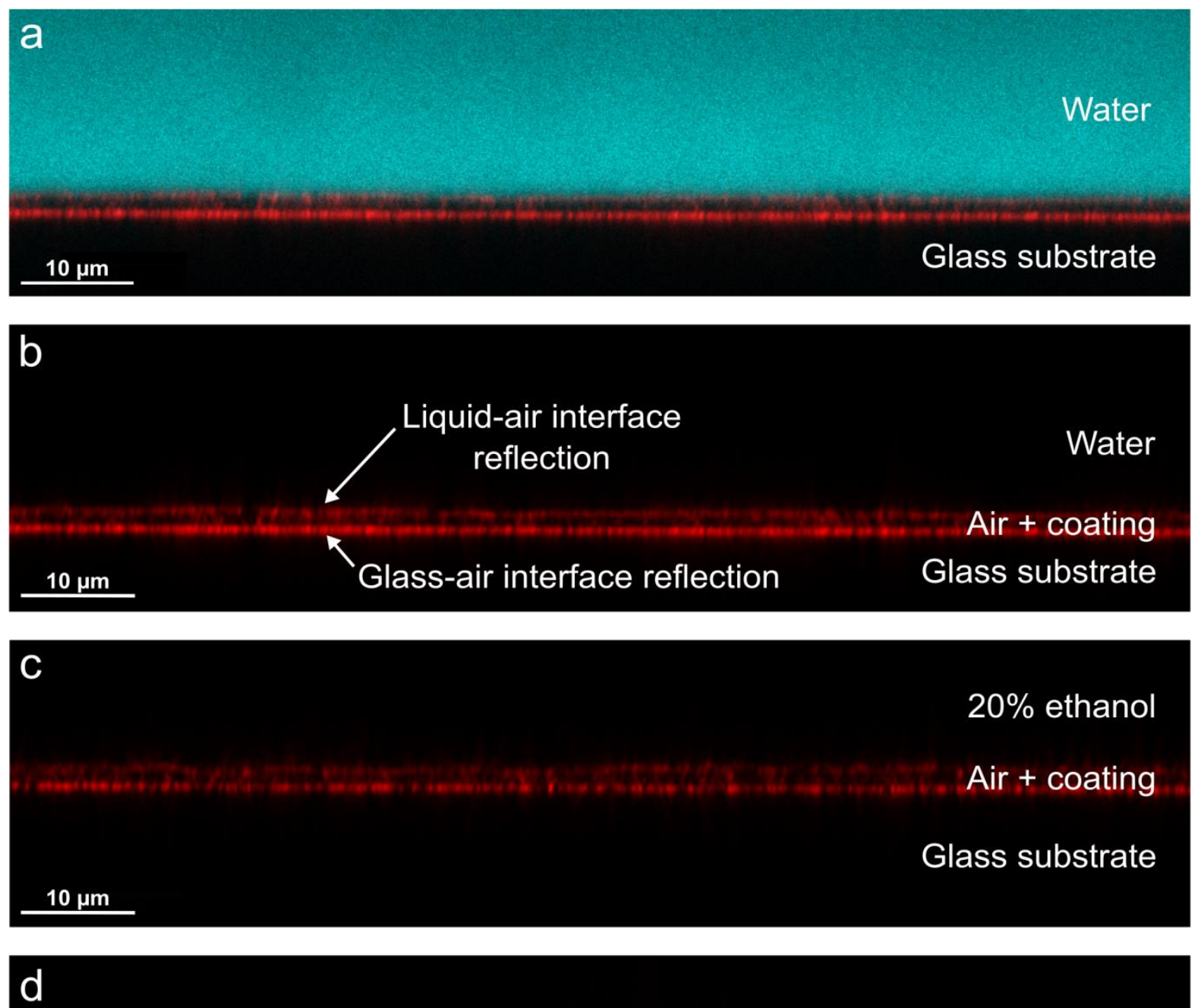

$40 \%$ ethanol

Glass substrate $10 \mu \mathrm{m}$

Figure S1. Laser scanning confocal microscopy images of liquids in a liquid cell on the superhydrophobic surface: (a) composite image of a water with fluorescent dye $\left(1 \mathrm{mg} \mathrm{L}^{-1}\right)$ in cyan and the light reflected from interfaces in red, (b) un-dyed water, (c) 20 vol\% ethanol, and (d) 40 vol\% ethanol. Scale bars $10 \mu \mathrm{m}$. 

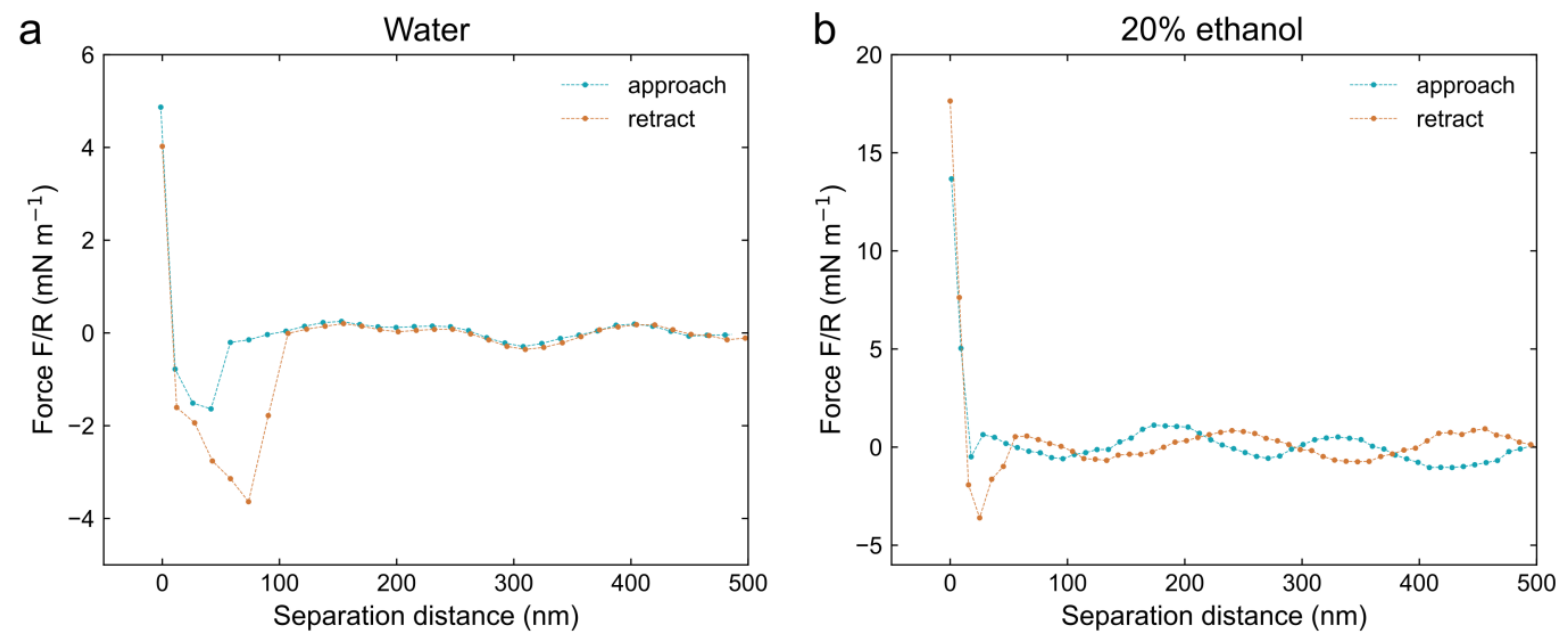

Figure S2. Examples of force curves showing interactions not consistent with an increase in gas capillary volume during separation between a superhydrophobic surface and a hydrophobic colloidal probe in (a) water and (b) 20 vol\% ethanol. 

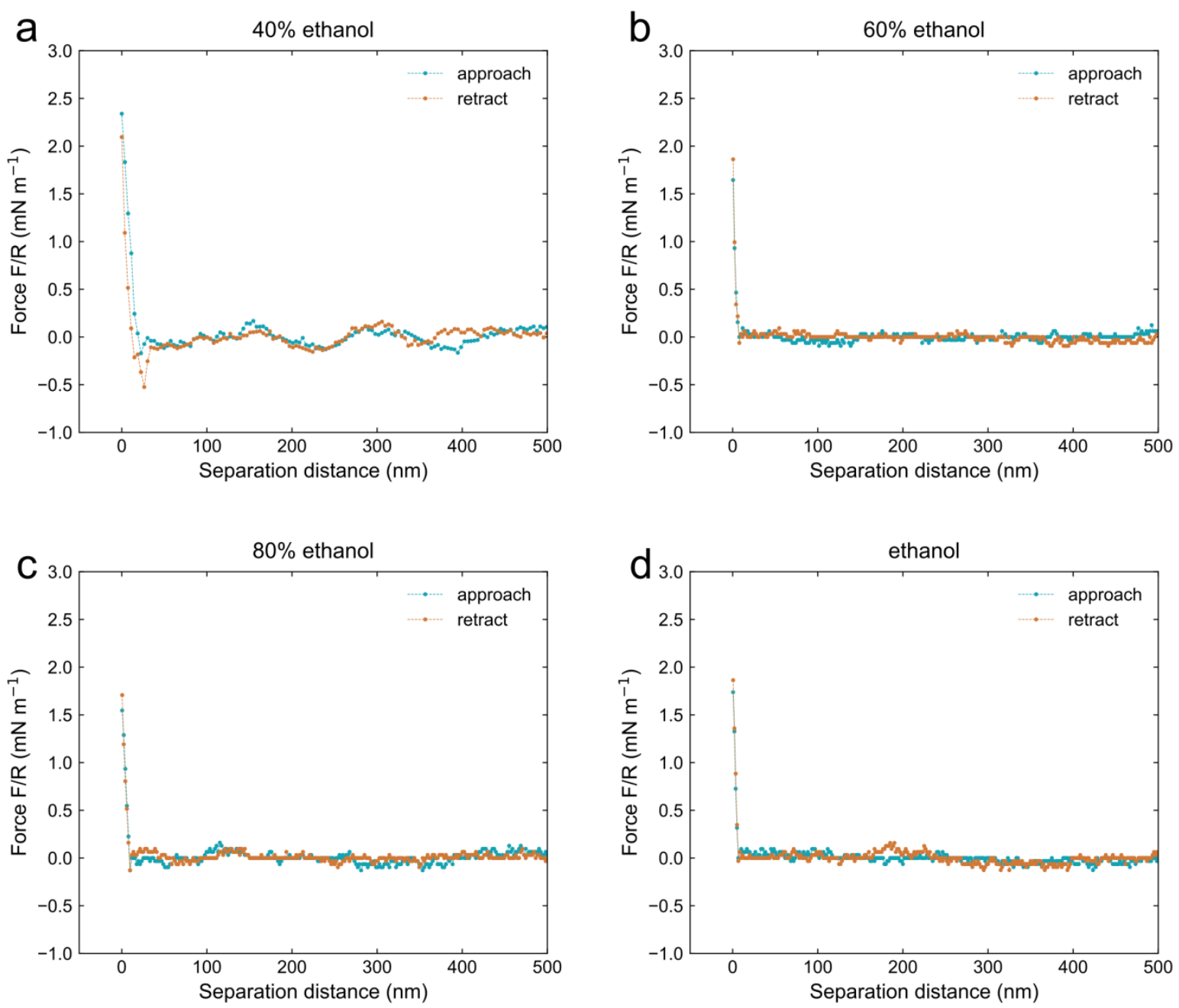

Figure S3. Typical force curves between a superhydrophobic surface and a hydrophobic colloidal probe in (a) 40 vol\% ethanol, (b) $60 \mathrm{vol} \%$ ethanol, (c) $80 \mathrm{vol} \%$ ethanol and (d) ethanol. 

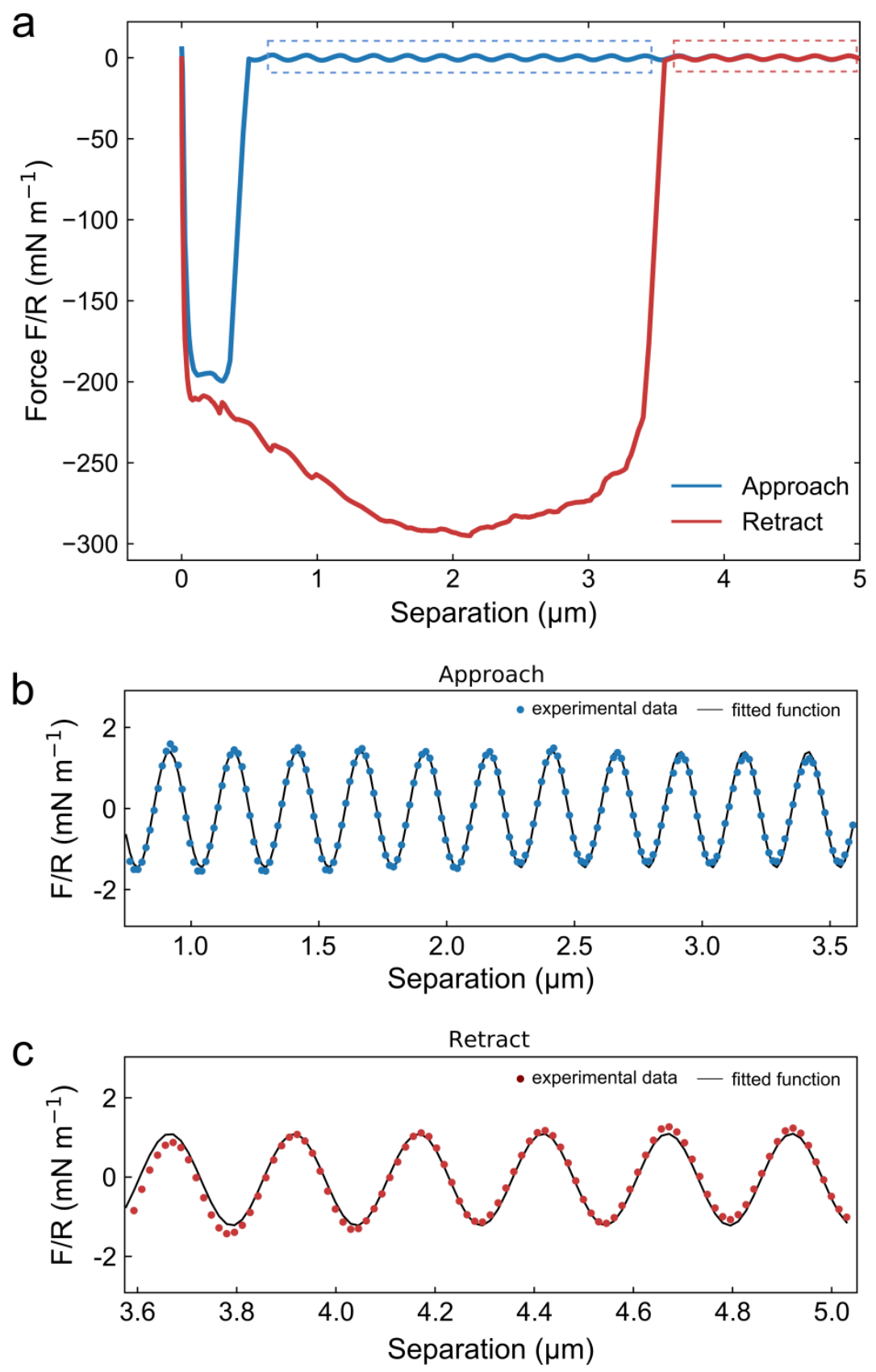

Figure S4. Example of force curve with interference pattern and fit to the sine wave equation (Eq. S4). 